International Conference on Recent Advances in Fluid and Thermal Sciences 2018

December 5-7, 2018 | Dubai, U.A.E.

\title{
Study of Interference Effects on Chimneys
}

\author{
K Shruti $^{1^{*}, \text { Sreeram Rajesh }}{ }^{2}$, Sabareesh GR ${ }^{2}$, PN Rao ${ }^{1}$ \\ ${ }^{1}$ Department of Civil Engineering, Birla Institute of Technology and Science- Pilani, \\ Hyderabad Campus, Telangana, India-500078 \\ ${ }^{2}$ Department of Mechanical Engineering, Birla Institute of Technology and Science- \\ Pilani, Hyderabad Campus, Telangana, India-500078 \\ *Email: shruti.konka@gmail.com
}

\begin{abstract}
.
Fluid flow studies play a vital role in proper assessing of wind loadson buildings and other civil engineering structures with various shapes. The presence of adjacent structure may alter wind loads on the structure of interest, by altering the fluid dynamics surrounding the structure. This effect is termed as interference effect and it depends on various factors such as terrain category, wind angle, geometry \& orientation of the structure, spacing between the structures etc. Thus, proper assessment of interference effect with above factors, can lead to efficient design of structures that can withstand the load variations due to the presence of interfering structures. The existing literature on wind interference effects, focuses more on experimental analysis of the problem, which is both expensive and time consuming. In this regard, computational fluid dynamics (CFD) approach can serve as a quicker and efficient tool. In an attempt to study the effect of geometry \&orientation of structures on interference phenomenon, turbulent flows around the buildings, cooling towers and chimneys have been simulated through CFD approach by performing Large Eddy Simulation (LES). In the present study, interference factors of a typical chimney structure has been evaluated by considering the presence of an identical chimney at varying distances, under different wind angles.
\end{abstract}

\section{Introduction}

Generally, the chimneys which are found at power plants or industries are designed as tapered structures with suitable height for the safe disposal of air pollutants from the flue gases into outside atmosphere without causing environmental hazards. Chimneys are often subjected to wind loads, earthquake loads, temperature loads along with normal dead loads and imposed loads [1]. However, in the present work, attention has been made only on the wind loads acting on chimneys in vicinity of an interfering chimney structure. Wind forces or pressures on the structure of interest may decrease/increase due to the presence of neighboring structure. This phenomenon is termed as interference effect. The chimney due to its long 
height and circular/tapered cross section, interacts with the flow around it. This flow around the structure is greatly disturbed due to the interfering structure, thus causing aerodynamic forces, vortex shedding phenomenon in some cases resulting in huge variations in the wind loads acting on the structure of interest. The height and cross section of the chimney structure are influencing factors of geometry to be considered for wind load analysis of structure. In addition to the above, factors such as terrain category, wind angle, geometry \& orientation of the structure, spacing between the structures etc., govern the interference phenomenon.

It is a common practice to perform flow analysis for the structure of interest considering only isolated structure. Even the codal provisions for design of chimneys are limited to isolated case only [2,3,4]. In a realistic scenario however, structures are found in groups such as buildings in a city, cooling towers in power plants and reactors, turbines in a wind farm, chimneys in factories etc. Such structures are usually analyzed for wind interference effects through wind tunnel experiments. As the experimental analysis of the problem is both expensive and time consuming, CFD approach and other numerical methods has evolved as an alternative.CFD approach can serve as an efficient tool to study this phenomenon and provide faster and cheaper solutions.

In the present study, attention has been given to simulate the turbulent flows around a chimney structure through CFD, by using LES model and evaluate the interference factors. The interference factor is defined as the ratio of mean pressure coefficient of principal structure in presence of interfering structure to the mean pressure coefficient of isolated principal structure [5,6]. The interference effect has been studied by considering the presence of an identical chimney tower at varying distances of 1.5D, 2D and $2.5 \mathrm{D}$, where $\mathrm{D}$ is the base diameter of the chimney. Variations in interference effect under different wind incident angles has also been addressed.

\section{Computational Model}

The geometry of the computational model consists of one principal chimney structure and another interfering chimney structure of same dimension arranged in tandem direction. The two cantilever type chimneys of height $H=0.28 \mathrm{~m}$, base and top diameters as $D=0.212 \mathrm{~m}$ and $D t=0.104 \mathrm{~m}$ respectively are considered. The $D / D t$ ratio is 2 . The distance between the two chimneys are maintained as $1.5 D, 2 D, 2.5 D$ respectively as shown in figure 1 . The two chimney structures are enclosed in a fluid domain of dimensions $X_{D} \times Y_{D} \times Z_{D}=2.52 \mathrm{~m} \times 1.8 \mathrm{~m} \times 2.2 \mathrm{~m}$. (Ref. figure 2.). The length of the domain is as per recommendations of J. Revuz et al., (2012), width and height of domain are as per wind tunnel dimensions of Wonsul Kim et.al., (2015). The fluid volume in the domain is modelled using finite volume method by creating a hexagonal structured grid. To obtain better accuracy with minimum computational time, the finer sub-grid mesh has been used for the region close to the structure as shown in figure 3 . The mesh has $5.5 \times 10^{-4} \mathrm{~m}$ as minimum grid size with $14,66,057$ cells in the domain. For simulation of atmospheric boundary layer, the mesh at the bottom portion of the geometric model is more refined up to 30 layers (slightly above building height) with a first layer thickness of $2.5 \times 10^{-3} \mathrm{~m}$ and a growth rate of 1.05 . The atmospheric boundary layer and turbulence characteristics are simulated using a user defined function (UDF), by performing LES for urban terrain category with exposure factor of $\alpha=1 / 4$ (as per AIJ codal principles). A mean wind velocity, $V$ of $8.2 \mathrm{~m} / \mathrm{sec}$ and turbulent intensity, $I$ of $20 \%$ at the chimney height is considered in the simulation. The computational scheme used in the present study has been validated for the two buildings case [7]. 

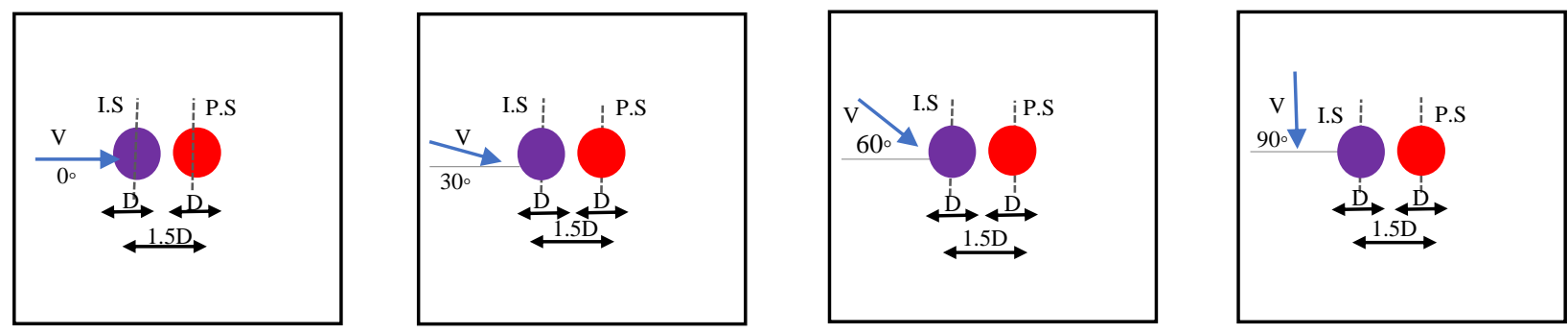

Figure 1. Typical arrangements of two chimneys in tandem direction under wind angles $0^{\circ}, 30^{\circ}, 60^{\circ} \& 90^{\circ}$

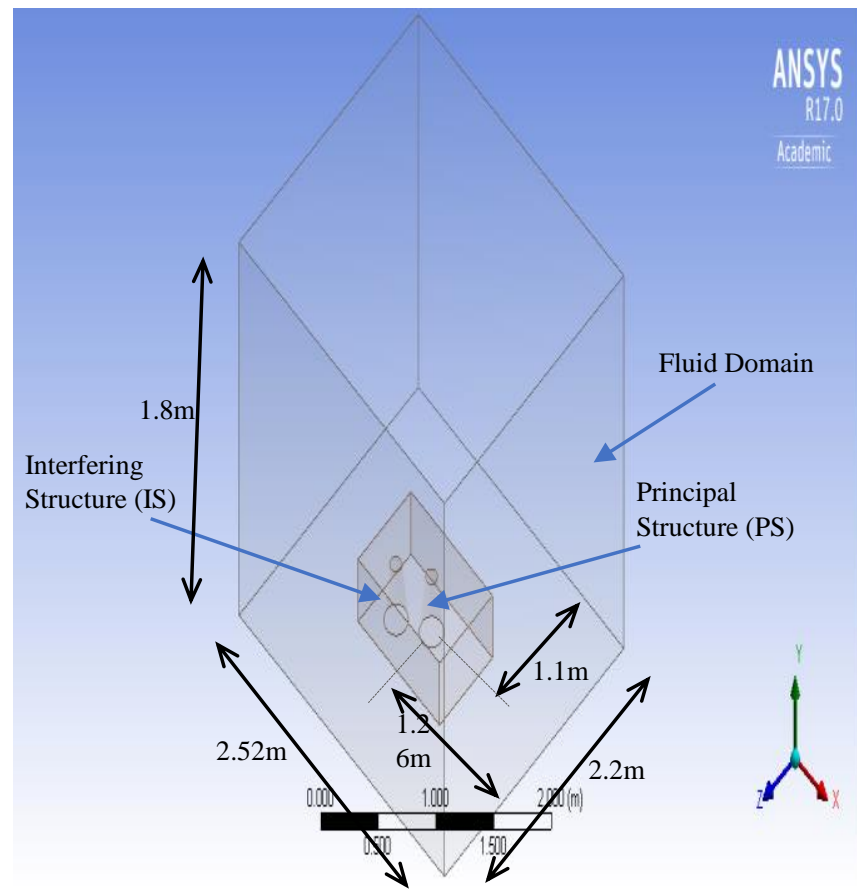

Figure 2. Geometry of Computational Model

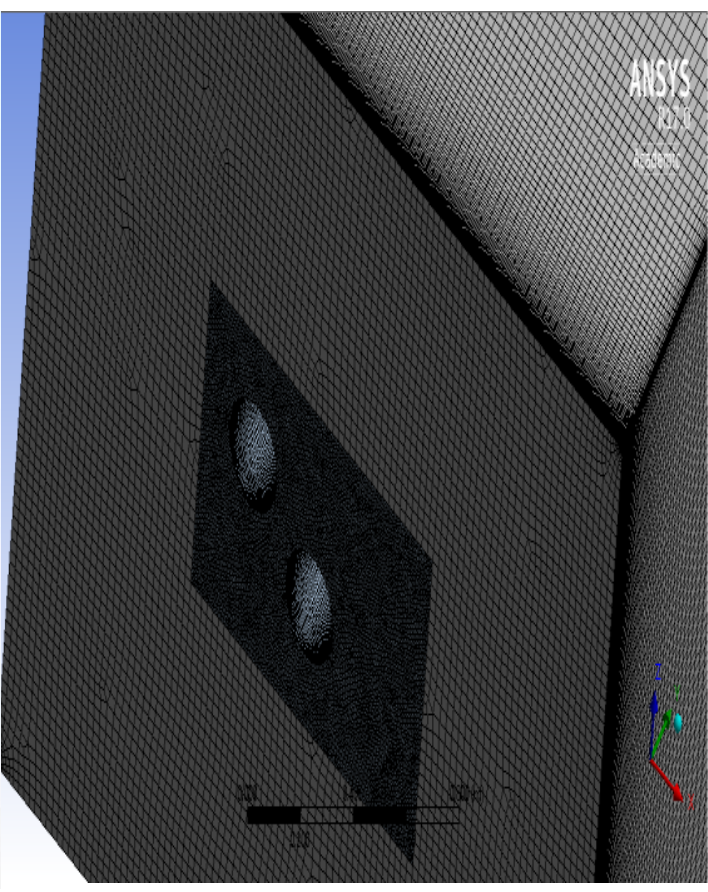

Figure 3. Computational Mesh

\section{Boundary Conditions}

The computational domain consists of a velocity inlet and pressure outlet as boundary conditions at inlet and outlet of the fluid domain. The top surface of the domain as zero shear wall and bottom surface of the domain as no slip wall boundary conditions are used with spire roughness height as $K s=0.00015 \mathrm{~m}$ (as per experimental data) to model the terrain under consideration. The side walls of the domain and the chimney walls are considered as no slip wall boundary condition. Turbulent atmospheric boundary layer is simulated at the inlet of domain through UDF using the standard power law wind profile as in Eq.1\&2. The incident turbulence intensity profile calculated from Eq.3is modelled with input parameters of $k$ and $\varepsilon$ (Eq.5) as per Blocken et.al (2007). The wind velocity profile and turbulent intensity profile used are based on measurements from experimental work of Wonsul Kim et.al (2015). 


$$
\begin{aligned}
& U_{Z}=U_{G}\left(\frac{Z}{z_{G}}\right)^{\alpha} \\
& U_{Z}=9.393\left(\frac{Z}{0.482}\right)^{\frac{1}{4}} \\
& I=0.1\left(\frac{Z}{z_{G}}\right)^{-\alpha-0.05} \\
& k=\frac{3}{2}\left(I . U_{\text {avg }}\right)^{2} \\
& \varepsilon=\frac{\left(U_{A B L}^{*}\right)^{3}}{K\left(y+y_{o}\right.} \\
& U^{*}=C_{\mu}^{\frac{1}{4}} k_{p}^{\frac{1}{2}} \\
& k_{s}=\left(\frac{9.793}{C_{k s}}\right) y_{o} \\
& \text { Where } C_{\mu}=0.09, K=0.41, y_{o}=7.6585 \times 10^{-6}
\end{aligned}
$$

\section{Numerical Scheme - Simulation}

Wind flow around the tall structures like chimneys exhibits turbulence characteristics. Presence of other identical structure in the vicinity of existing structure may further change the flow dynamics surrounding the structure. Such wind interference effects are to be modeled accurately by adopting suitable numerical scheme through proper modelling of turbulence characteristics of incident wind flow. Thus, transient simulations are performed using above mentioned boundary conditions to model this unsteady turbulent flow. LES with Smagorinsky-Lilly SGS model without dynamic stress option is used to perform these transient simulations. In this model, filtered forms of continuity equation and Reynolds Averaged Navier Stokes (RANS) equation [8] present in the governing equation are to be solved using the applied boundary conditions. Simulation is initialized at the inlet. SIMPLE Pressure velocity coupling algorithm is used as solver. Initially, steady state simulations were performed using realizable k- $\varepsilon$ model with standard wall functions to obtain the converged solution. Subsequently, transient simulations using LES were turned on after obtaining the converged solution and the simulations were carried out with $0.001 \mathrm{~s}$ as time step size and 150 time steps. Second order upwind scheme with bounded second order implicit transient formulation is used. The convergence criteria is set to 0.0001 for all parameters.

\section{Results and Discussions}

The numerical scheme used for the present study has been validated by K. Shruti et al (2018) with respect to experimental results of Wonsul Kim et al (2015). This validated numerical scheme is extended to present chimney case.

The mean pressure coefficients of principal chimney structure at a height of $0.228 \mathrm{~m}$ are computed for both interference case and isolated case. Interference factors (I.F) are evaluated using Eq. 8, by varying the locations of interfering chimney with respect to principal chimney as $1.5 D, 2 D$ and $2.5 D$ for different wind angles $0^{\circ}, 30^{\circ}, 60^{\circ} \& 90^{\circ}$

$$
\text { I. F }=\frac{\text { Mean Pressure Coefficient of Principal chimney structure in presence of Interfering structure }}{\text { Mean Pressure Coefficient of isolated chimney structure }}
$$


I.F value equal to one is treated as an ideal case without having any interference effect (resembling as that of isolated case). I.F value less than one represents shielding effect and greater than one represents enhanced wind load[9]. Interference effects of two chimneys configuration are assessed by plotting I.F values against $\mathrm{s} / \mathrm{b}$ ratio for different wind angles and different interfering distances as shown below in Figures 5 to 7. Here, the arc length along the periphery of the structure from the wind incident point is taken as $s$ and the chimney diameter at a height of $0.228 \mathrm{~m}$ is taken as $b(=0.12 \mathrm{~m})$, which is shown in Figure 4.

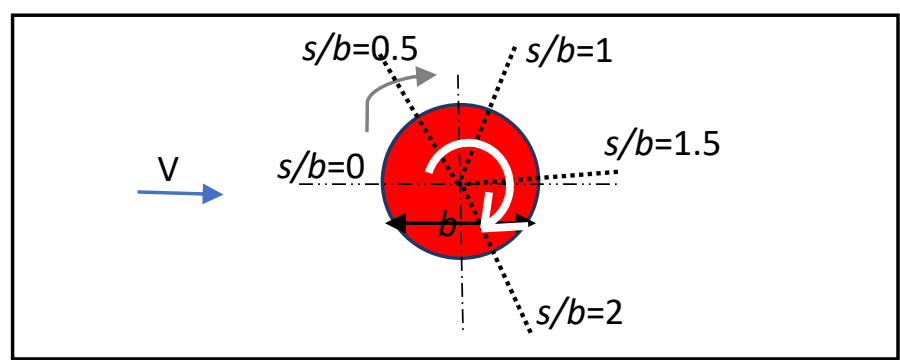

Figure $4 s / b$ ratio representation

\section{Interference effects on chimneys separated by $1.5 \mathrm{D}$ distance}

When interfering chimney is placed at a distance of $1.5 \mathrm{D}$ from the principal structure (in tandem direction), I.F values plotted against $\mathrm{s} / \mathrm{b}$ ratios for different wind angles are shown in below Figure5. From the below figure, it can be inferred that when the structure is subjected to $0^{\circ}$ wind angle, shielding effect is observed on the principal structure for $s / b$ ratios ranging from 0 to 2 with maximum shielding upto $49 \%$ among all wind angles. The maximum $I . F$ values are noticed in between an $s / b$ ratio of 0.6 to 1.4 (right portion of leeward face) for all wind angles while maximum I.F value of 1.74 is observed for $90^{\circ}$ wind angle at an $s / b$ ratio of 0 . An increasing trend in variation of interference effect is observed from $s / b$ ratio of 0.6 to 1 for all wind angles except $0^{\circ}$ (which is having maximum I.F value of 0.89 at $s / b$ of 0.6 ). The structure when incident with a wind angle of $90^{\circ}$, exhibits a variation of the order of $8-74 \%$ in I.F.

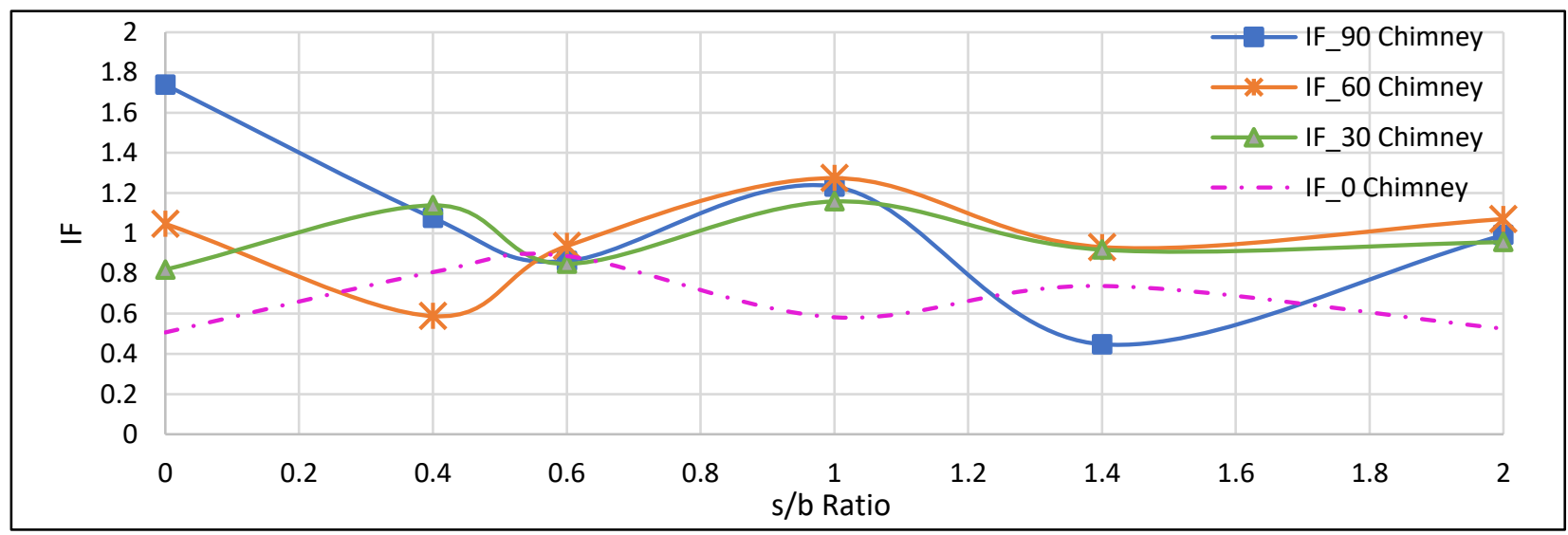

Figure5. Interference factors against $s / b$ ratio for interfering distance of $1.5 D$ 


\section{Interference effects on chimneys separated by $2 D$ distance}

When interfering chimney is placed at a distance of $2 D$ from the principal structure (in tandem direction), I.F values are plotted against $s / b$ ratios for different wind angles as shown in Figure.6. It can be observed from the figure that, the peak I.F values with maximum of 58\% are from $s / b$ ratio of 0 to 0.6 (left of windward face) for all wind angles except for 30. Majority of the shielding effect is observed at an $s / b$ ratio of 0.8 to 1.4 for $0^{\circ}$ and $30^{\circ}$ wind angles. However, $0^{\circ}$ wind angle shows maximum percentage of shielding upto $57 \%$ at $s / b$ ratio of 2 . At $60^{\circ}$ and $90^{\circ}$, there is only a slight variation in $I . F$ values for $s / b$ ratio greater than 1 and the interference effect is almost negligible for $s / b$ ratio greater than 1.4 for all wind angles except at $0^{\circ}$ where the shielding effect is predominant.

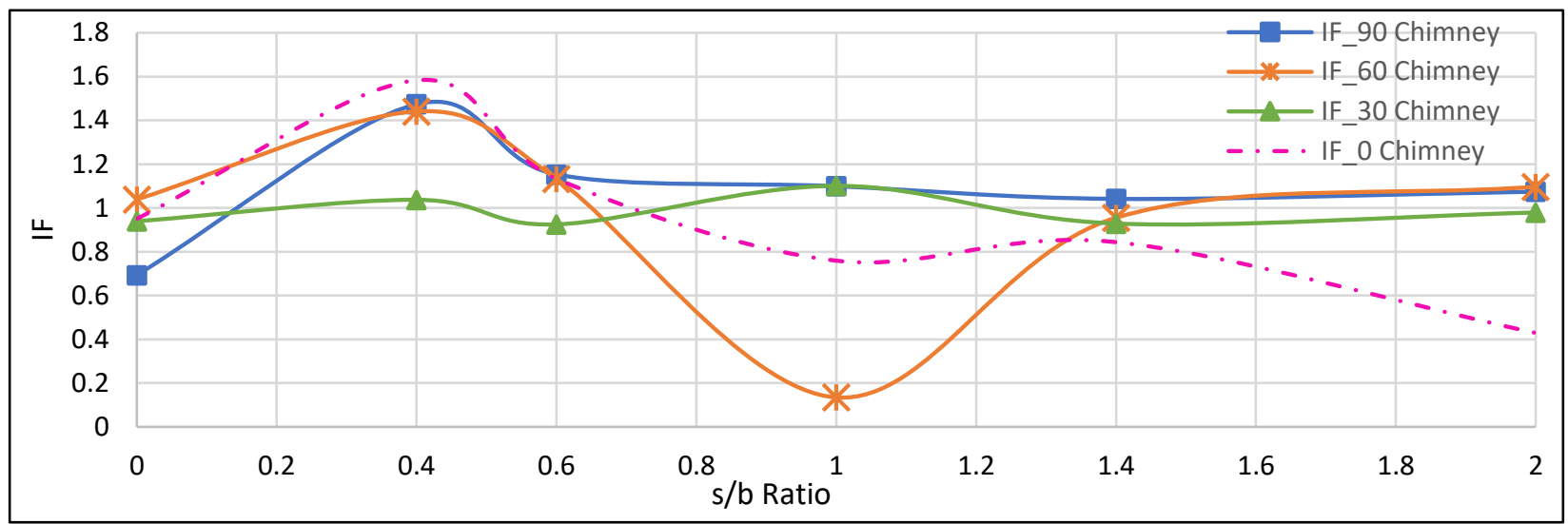

Figure 6. Interference factors against $s / b$ ratio for interfering distance of $2 D$

\section{Interference effects on chimneys separated by $2.5 \mathrm{D}$ distance}

When interfering chimney is placed at a distance of $2.5 \mathrm{D}$ from the principal structure (in tandem direction), I.F values plotted against $\mathrm{s} / \mathrm{b}$ ratios for different wind angles are shown in below Figure.7. It is observed that, when the structure is subjected to $0^{\circ}$ wind angle, shielding effect is observed on principal structure for all s/b ratios ranging from 0 to 2 with maximum shielding effect of $50 \%$ at $s / b$ of 0 . The peak $I . F$ values are at $s / b$ ratio from 0.4 to 1 (left portion of windward face) for all wind angles with higher interference effect upto $39 \%$ at $\mathrm{s} / \mathrm{b}$ of 1 . The $I . F$ values are comparatively higher at $s / b$ less 1 than at $s / b$ greater 1 for all wind incident angles. The structures subjected to $30^{\circ}$ and $90^{\circ}$ wind angles shows similar trend of variation of $I . F$ values for $s / b$ ratio from 1 to 2 .

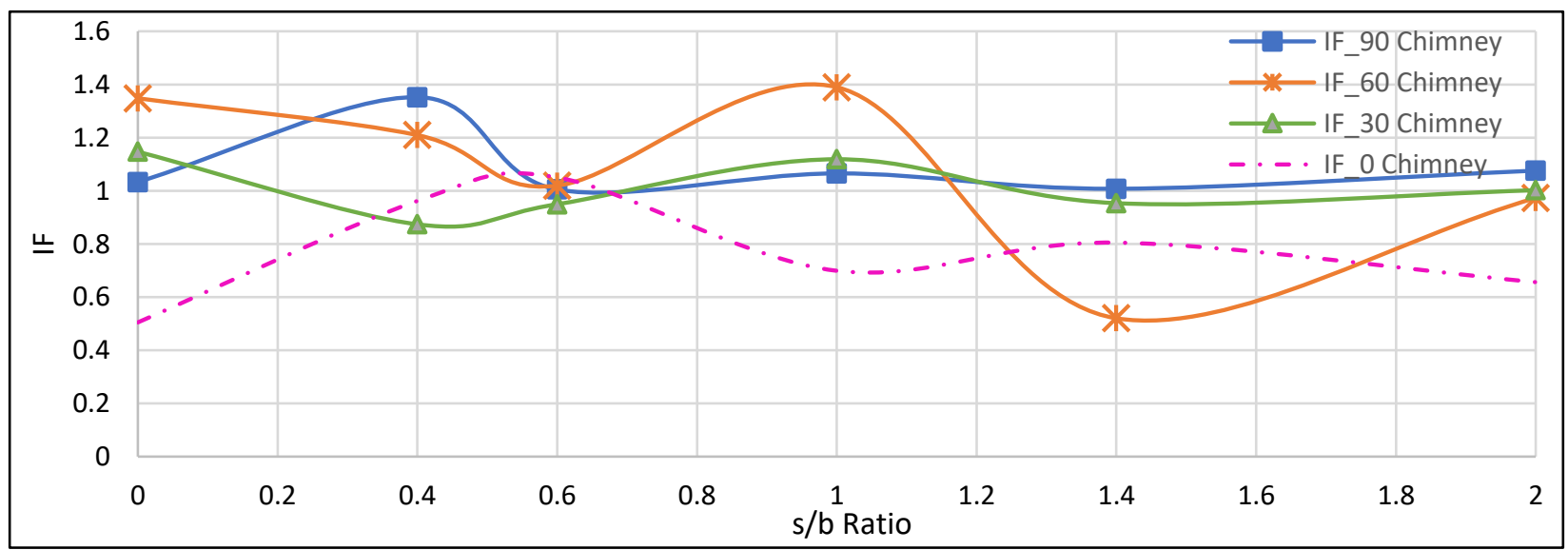

Figure 7. Interference factors against $s / b$ ratio for interfering distance of $2.5 D$ 


\section{Conclusions}

The results of interference effects of two chimney structures have been evaluated for different locations of interfering chimney and different wind angles through CFD approach and the following observations were made:

(1) Approximately $40-50 \%$ of the shielding effect observed for all locations of interfering structure considered in the present study is with a $0^{\circ}$ wind incident angle.

(2) Shielding effect increases as $\mathrm{s} / \mathrm{b}$ ratio approaches 1 for the chimney located at $2 D$.

(3) $30^{\circ}$ wind incident angle has the least interference effect among all wind angles for chimney configurations considered in the present study.

(4) The regions with $s / b$ from 0 to 0.6 (left windward face) of principal chimney structure is subjected to an enhanced wind loads for all chimney locations $(1.5 D, 2 D, 2.5 D)$ and for all wind angles $\left(0^{\circ}, 30^{\circ}, 60^{\circ}\right.$, $\left.90^{\circ}\right)$.

(5) The primary structure experiences peak load (maximum interference) for the configuration: $1.5 D-90^{\circ}$ and greatest shielding for the configuration: $2 D-60^{\circ}$.

Thus, significant aspects regarding critical face of the chimney structure such as location of the interfering chimney structure and wind angle which are subjected to high interference effects are identified through present study.

\section{References}

[1] Nishant Sudesh Jadhav, R S Talikoti2017Analysis of Self-Supporting Steel Chimney using ANSYS Software Int., J. for Scientific Research \& Development 52321-0613

[2] IS 6533-1 (1989): Code of practice for design and construction of steel chimneys, Part 1: Mechanical aspects [CED 7: Structural Engineering and structural sections]

[3] IS 6533-2 (1989): Code of practice for design and construction of steel chimneys, Part 2: Structural aspects [CED 7: Structural Engineering and structural sections]

[4] IS: 875 (Part 3) (2011): Wind Loads on Buildings and Structures- Commentary Document No.: IITKGSDMA-Wind 02-V 5.0

[5] Ming Gu, Zhuang-Ning Xie 2008 Interference effects of tall buildings under wind action Proc., of

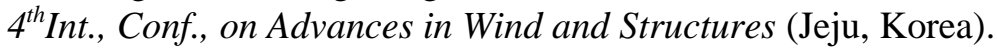

[6] A C Khanduri, T Stathopoulos, C Bedard 1998 Wind-induced interference effects on buildings - a review of the state-of-the-art J. Eng. Structures Vol20. No.7 pp.617-630

[7] K.Shruti, Sabareesh G R, P N Rao 2018 Mitigating Wind Induced Disasters on a group of Buildings and Cooling Towers due to Interference EffectProc.,of Int., Workshop on Wind Related disasters and mitigation(Sendai , Japan).

[8] Tetsuro Tamura 2009 Large eddy simulation on building aerodynamics The 7th Asia Pacific Conf., on Wind Engineering (Taipei, Taiwan).

[9] Theodore Stathopoulos 1984 Adverse wind loads on low buildings due to buffeting Journal of Structural Engineering ASCE 110(10) 2374-2392.

[10] Wonsul Kim, Yukio Tamura, AkihitoYoshida2015Interference effects on aerodynamic wind forces between two buildings J. of Wind Eng. Ind. Aerodyn.,147 (2015)186-201

[11] Julia Revuz 2011Numerical simulation of the wind flow around a tall building and its dynamic response to wind excitation [Ph.D Dissertation] Univ. of Nottingham.

[12] AIJ (2004). RLB Recommendations for Loads on Buildings, Tokyo, Structural Standards Committee.

[13] Bert Blocken, Jan Carmelieta, Ted Stathopoulos 2007 CFD evaluation of wind speed conditions in passages between parallel buildings-effect of wall-function roughness modifications for the atmospheric boundary layer flow J. of Wind Eng. and Ind. Aerodyn., Vol. 95, pp.941-962 
[14] Mohd. Mohsin Khan, Amrit Kumar Roy2017 CFD simulation of wind effects on Industrial RCC chimney Int., J. of Civil Engineering and Technology Vol 8 Issue 1 pp. 1008-1020

[15] T Saran Kumar, R Nagavinothini 2015 Wind Analysis and Analytical Study on Vortex Shedding Effect on Steel Chimney using CFD Int., J. of Science Eng. Tech. and Research Vol 4 Issue 4 ISSN:2278 $-7798$

[16] Jisha S V, B R Jayalekshmi, R Shivashankar2012Across Wind Response of Tall Reinforced Concrete Chimneys Considering the Flexibility of SoilInt., J. of Eng. Research and Tech. Vol 1 Issue 8 ISSN: 22780181 